\title{
1. Introduction: Dealing with uncertainty in environmental decision making
}

\subsection{INTRODUCTION TO THE PROBLEM OF ENVIRONMENTAL UNCERTAINTY}

On the whole, men are more good than bad; that however isn't the real point. But they are more or less ignorant, and it is this that we call vice or virtue; the most incorrigible vice being that of an ignorance that fancies it knows everything and therefore claims for itself the right to kill.

Albert Camus, The Plague (1972)

At the core of this book is the assertion that many decisions surrounding the environment are conditioned by the presence of uncertainty. The recognition that there are a number of different modes of uncertainty radically alters the way in which environmental uncertainty can be dealt with both at a epistemological and practical level, and necessitates an alternative approach to that encompassed by traditional probability-based models. However, while there is an extensive literature on probability-based models of risk in decision making (Perrings et al., 1995), the literature on dealing with environmental uncertainty and in particular with what will be termed 'hard uncertainty' ${ }^{1}$ in decision making is rather limited. It is this issue of dealing with environmental uncertainty in decision making that provides the impetus for the research carried out and presented in this book.

The uncertainty that decision makers are faced with in many environmental problems is derived from three main sources. First, uncertainty exists about the resilience of ecosystems and the consequences of human induced change for ecosystems, particularly when the action could result in a ecosystem threshold being exceeded. Second, uncertainty is often present with regard to the value of those changes. The third source stems from uncertainty as to future supply of vital ecosystem functions and services that may be of unanticipated importance in terms of human welfare. The prevalence of uncertainty in environmental problems raises questions about how uncertainty is and should be evaluated in the decision-making process.

While current models can claim some virtues in dealing with risk or what will be termed soft uncertainty, ${ }^{2}$ it is argued that they are limited in terms of their applications to hard uncertainty problems of the sort often encountered 
on a regular basis in environmental decisions. A central hypothesis of this book is therefore that decision models based on risk or soft uncertainty and in particular the notion of probability cannot be expected to deal either adequately or explicitly with issues of environmental uncertainty such as biodiversity loss. Given the need for an alternative and novel decision-making framework for dealing with environmental uncertainty, the Shackle model will be proposed and interpreted in the context of environmental uncertainty. The question of whether this model is useful in explaining the way in which decisions are made under environmental uncertainty will be addressed. In addition a key element of the research aims to assess the extent to which the Shackle model can be adapted to serve as a decision-making framework which allows explicit attention to be given to uncertainty.

The research therefore aims to provide a contribution in five main areas. First, an important step will be in developing criteria for defining the nature and the different modalities of environmental uncertainty, and for assessing the implications of the different levels of uncertainty in terms of decision making. Second, on the basis of these criteria traditional models for dealing with risk will be critically assessed in terms of their suitability for dealing with environmental uncertainty. Third, an alternative model for dealing with uncertainty based on the work of George Shackle will be interpreted and applied in relation to environmental uncertainty, and fourth, the practical application of a model that will aid decision makers to deal adequately with uncertainty will be advanced.

The final contribution centres on a reassessment of how we rationalize decision making when faced with the realities of uncertainty. The interpretation of the resulting model of uncertainty necessitates a departure from the use of the traditional criteria used to assess decision rules in terms of their rationality. Consequently models of environmental uncertainty need to be assessed more in terms of their procedural rationality and their application at the ex ante stage of decision making rather than purely in terms of their optimality or usefulness in providing ex post explanations of human behaviour in decision making.

In attempting to deal with the realities of uncertainty in environmental decision making, a practical as well as theoretical approach has been employed. It was this reason that motivated the use of a case study to apply the Shackle model of decision making in a real world experiment. The fieldwork and subsequent data that were collected focused on the way that uncertainty about the environmental effects of development projects was dealt with by a developing country, Belize, and by the Inter-American Development Bank. The interviews conducted allowed the practical application of an alternative model of uncertainty, as well as providing valuable observations on the more general nature of dealing with uncertainty in development agencies such as 
the Inter-American Development Bank, the British Development Division in the Caribbean and the World Bank. The quantitative and qualitative data collected have also provided a useful insight into questions surrounding whether or not the Shackle model is useful in explaining the way that decision makers deal with uncertainty. To the author's knowledge this is the first major application of the Shackle model in the context of environmental uncertainty ${ }^{3}$ and is the first application to a real world decision rather than a laboratory experiment.

Before dealing with the problem of uncertainty in environmental decision making, however, it is necessary to outline the research framework that is chosen, what such a framework implies and the theoretical issues that will need to be addressed. Consequently the rest of this chapter will be concerned with positioning this research within the overall context of sustainable development and biodiversity. The first part of the chapter will be concerned with some of the theoretical issues that sustainability and biodiversity pose for this research. After a brief outline of some of the origins and the main issues posed by sustainability, the notion of the interconnectedness between the ecological and economic systems will be highlighted. Having a greater understanding of key characteristics of this system is important in the debate regarding the form of sustainability advocated, and in particular in emphasizing the difficulty in assuming the substitutability of natural and other forms of capital. The key issue of the uncertainty raised due to the public good nature of many environmental services will also be reviewed and the problem of irreversibility and the appropriateness of equilibrium analysis tackled. The implications of the precautionary principle in the context of sustainable development will be briefly considered, before a framework for research is outlined and the main hypotheses to be addressed are formulated.

\subsection{INTRODUCTION: SUSTAINABLE DEVELOPMENT}

The problem of uncertainty in environmental decision making stems from growing concerns about the impact of human activity on the environment, encapsulated in the ever growing discourse on sustainable development. The significance of this debate for the research is that it makes the issue of dealing with environmental uncertainty in decision making imperative and it is within the context of meeting sustainability objectives that the problem of environmental uncertainty will be addressed. Understandably the complexity of the relationship between humans and their environment has produced a wide set of interpretations of sustainability, all with a number of either implicit or explicit implications that merit careful consideration. However sustainability is defined, it will inevitably involve making trade-offs between a number of 
concerns, which are often competing. It is the making of the trade-offs and the role that uncertainty plays in some of the main concerns of sustainability that form the focus of this chapter.

\subsubsection{The Research Context: Sustainable Development and Biodiversity}

With the publication of the Bruntland Report in the 1980s the sustainability discourse came to the fore of the world political agenda (Common, 1995). The report tried to reconcile some of the earlier development failures based purely on economic growth with a development paradigm in which social and inter-generational objectives were recognized as distinct and were given equal weight to economic efficiency, and in which the long-term degradation of the environment was seen as a barrier to development (Munasinghe, 1993b). Thus, according to the report: 'Sustainable Development seeks to meet the needs and aspirations of the present without compromising the ability to meet those of the future' (WCED, 1987).

Inherent in the concept of sustainable development is therefore an ethical condition that future generations have a right to have handed on to them the capacity to generate for themselves a level of well-being no less than that enjoyed by the current generation (Barbier et al., 1995). Another important issue is whether at present rates of consumption it is indeed possible for economic growth and sustainability to go hand in hand. For Herman Daly it is crucial to distinguish between development in terms of qualitative change and growth which is defined purely in quantitative terms (Daly and Cobb, 1989). What is most important is that the sustainable development debate represents an attempt to focus on the nature and type of economic growth that would be compatible with sustainable development and on how traditional development objectives could be reconciled with concerns for environmental quality and inter temporal equity.

The promotion of environmental concerns in the sustainable development discourse is itself perhaps best manifested in the increasing prominence given to biodiversity. One of the first calls for this link can be seen in the First World Conservation Strategy which argued that:

the maintenance of essential ecological processes and life support systems, the preservation of genetic diversity, and the sustainable utilisation of species and ecosystem have the overall aim of achieving sustainable development through the conservation of living resources. (IUCN/ UNEP/ WWF, 1980 in Barbier et al., 1994)

The issue of biodiversity gained international recognition as a result of the Biodiversity convention signed under the auspices of the UNCED at Rio in 
June 1992. The concept of biodiversity involves a complexity of meanings, although all imply some measure of distance (Barbier et al., 1995). Based on this principle, three main perspectives can be seen. The first is that of genetic diversity, which refers to the heritable variation within and between populations, or the sum of the genetic information contained in the genes of plants and micro-organisms. The second is that of species diversity, which refers simply to the number of species in a particular habitat. The third, ecosystem diversity, refers to the diversity both within and between ecological processes, such as habitats, biotic communities and ecological processes (such as photosynthesis, nutrient cycling and nitrogen fixing) (Blaikie, 1996) and is based on the concept of the functional role of species in supporting critical structuring processes. Arguably it is this concept of functional diversity that is the most crucial to the sustainable development debate. Unfortunately it is more difficult to classify than the other categories of diversity. Functional diversity can be defined in two main ways: first in terms of the diversity of ecological functions performed by different species; and second in terms of the diversity of species performing a given ecological function (Barbier et al., 1995).

A rationale exists therefore for linking biodiversity and the sustainable development discourse on scientific, economic and moral grounds, in that biological diversity is the underlying component in our life support system, that biological diversity provides us with many direct and indirect economic benefits and that all species require respect regardless of their use in humanity. As will be highlighted in this and future chapters, the large degree of uncertainty inherent in decisions regarding biodiversity, means that the issue merits particular attention in the context of environmental uncertainty.

However operationalized, the concept of sustainability necessarily involves making a number of trade-offs between, for example, ecological objectives such as the maintenance of biodiversity, economic objectives such as economic growth, and social cultural objectives such as wealth distribution. Figure 1.1 highlights some of the trade-offs that may be required to be met, with the boxes contained in the middle of the arrows indicating issues that need to be resolved in order to facilitate the making of trade-offs. The context for this book is restricted, however, to focusing on the uncertainty which arises as a result of the interactions between the highly interconnected and complex economic and ecological system, and the accompanying trade-offs between the economic and ecological objectives, within sustainable development. ${ }^{5}$ Inevitably, especially when the role of uncertainty in decision making is considered, socio-cultural and political issues will be raised, but due to the scope of these issues, the bulk will remain outside the consideration of this book. 


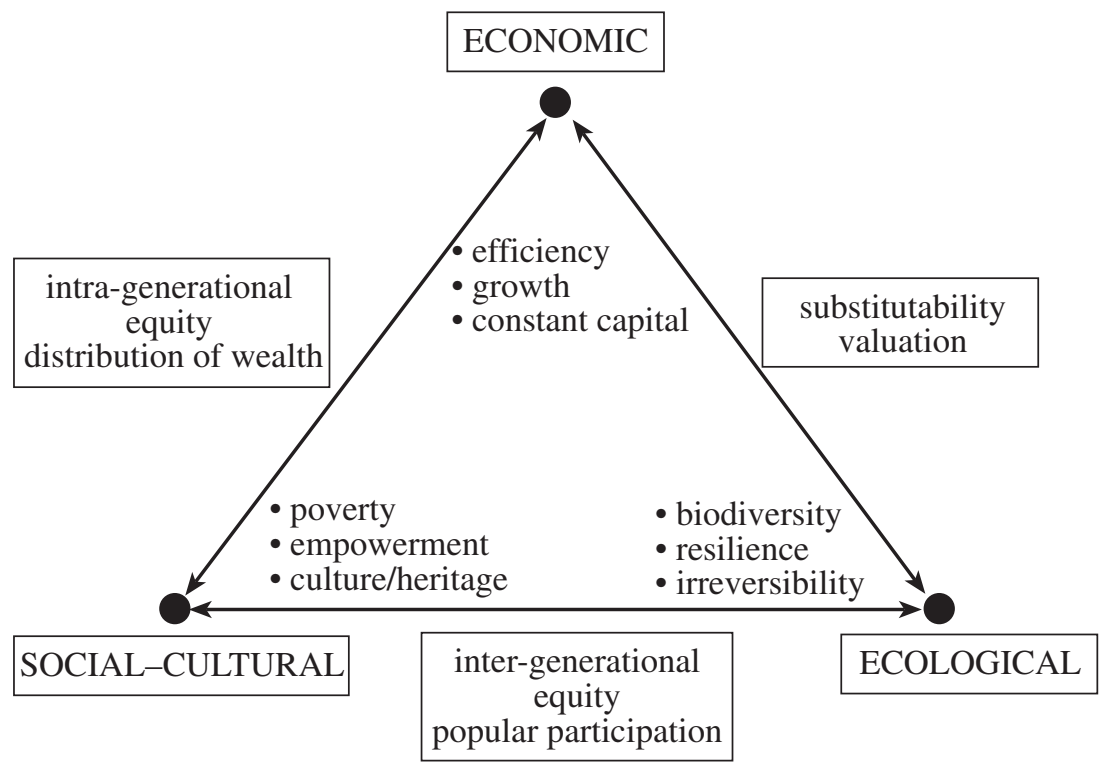

Figure 1.1 Trade-offs among the three main objectives of sustainable development ${ }^{6}$

\subsection{SUSTAINABLE DEVELOPMENT AND UNCERTAINTY: KEY ISSUES}

One of the pivotal arguments in this book is that uncertainty is central to the sustainable development discourse and will need to be addressed if sustainability objectives are to be met. Indeed given certainty about the future, the attainment of sustainability goals would arguably be relatively straightforward. The next section considers some of the most important uncertainty issues raised by the sustainable development discourse. As will be seen, it is in stressing the importance of these issues within the sustainability debate that such a potentially explosive mix for environmental decision making is implied (Facheux and Froger, 1995).

\subsubsection{The Complexity and the Interconnectedness of the Ecological- Economic System}

While academically it may be more convenient to analyse the economic and ecological systems separately, increasingly many environmental and ecological economists have begun to emphasize the fact that the economic system is both 
highly connected with and dependent on the ecological system. Increasing evidence points to linkages between economic activity and the environment which are pervasive and complex. The more highly connected ecological and economic systems are, the more change in one implies change in the other, or as Norgaard (1984) terms it, the more they 'coevolve'. The implication of this is that it is necessary to look at the economy not as a closed system but as a component of a wider system. Thus matter and energy used up in human production and consumption activities must eventually end up in environmental systems and result in significant forms of environmental and economic damage, such as pollution, (Perman et al., 1996). Furthermore the entropic nature of the system ensures that, while the total amount of mass or energy remains the same, its structure does not. The consequence of this is that economicecological interactions may lead to irreversible losses (see Section 1.3.5).

The recognition of the possibility that there may in fact be limits to the impacts of economic activity on the environment is based on the realization that environmental resources provide a number of both direct and equally crucial indirect services and functions which do in fact affect human welfare. Moreover, it can be argued that the regulatory functions performed by ecosystems, such as waste assimilation, indirectly support all economic activity (Barbier et al., 1995). The processes among and within the physical, biological and chemical components of an ecosystem result in specific types of ecosystem function or property, such as nutrient cycling, biological productivity, hydrology and sedimentation. These interactions or life support functions in turn generate many ecological resources and services that are of fundamental value in that they sustain human societies (see Table 1.1).

The highly interconnected nature of the economy and of the environment and the complex series of feedbacks between the two, further complicate the sustainability problem. Thus meeting an ecological objective such as the maintenance of key ecological functions and services is complicated by the uncertainty surrounding the effects of increased economic activity on these ecological functions. This fundamental issue of uncertainty boils down to the problem of ecosystem thresholds and resilience. It is sufficient to remember that the effects of economic activity have very real consequences for the environment, which due to the complexity of the interactions pose considerable problems in defining quantitatively the extent and type of economic activity that is consistent with ecological sustainability. As Michael Common argues:

the level of human impact on the national environment is now such that its capacity to support future economic activity at the level required by the human population and its aspirations is questionable. The issues arising are characterised by ignorance and uncertainty. (Common, 1995: p. 4) 
Table 1.1 Ecosystem functions and their uses in the economic system

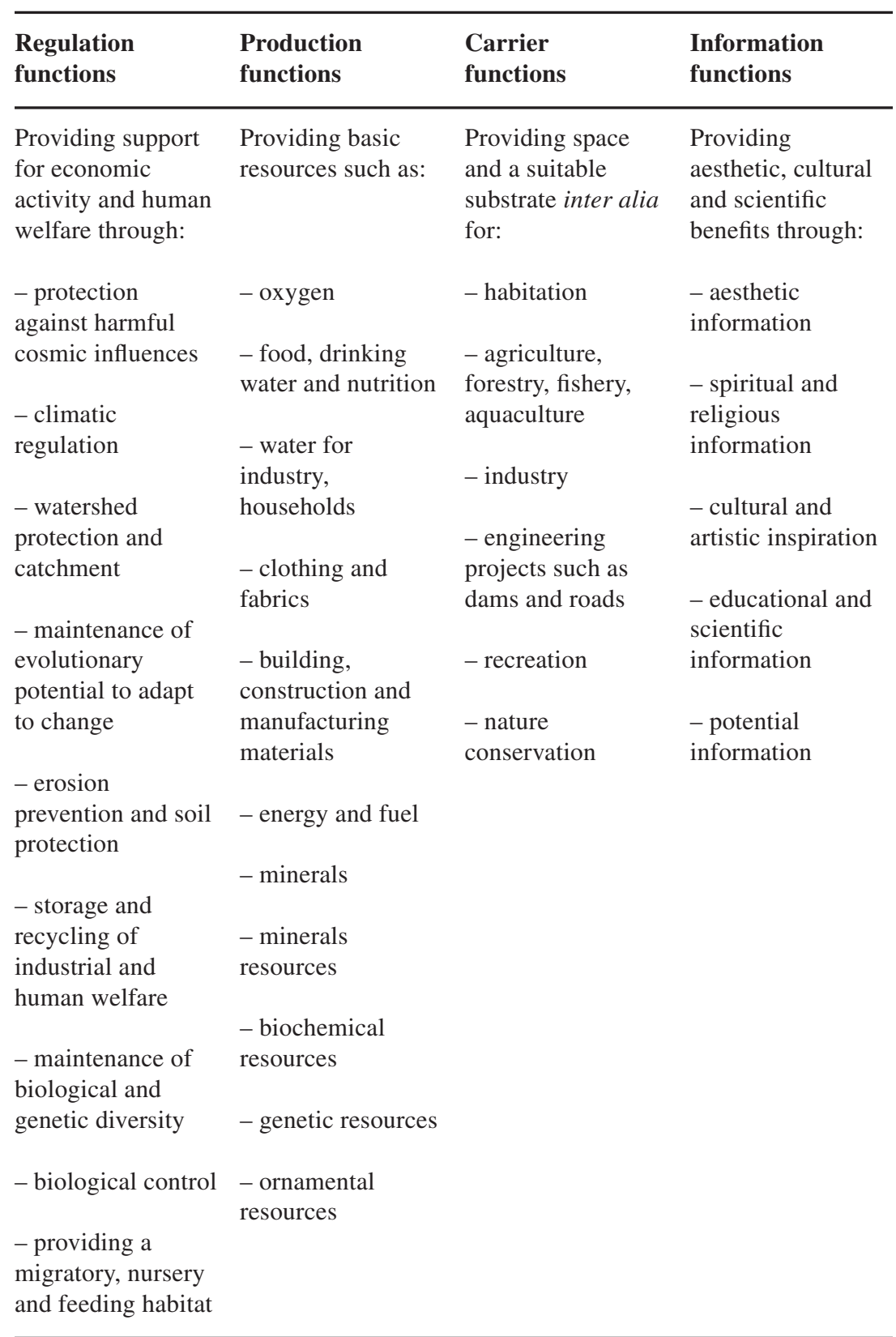

Source: Adapted from Barbier et al., 1995 
Although ecosystems contain hundreds and thousands of species interacting amongst themselves and with their physical system, the consensus is that the system is driven by a relatively small number of biotic and abiotic variables, on whose interactions the overall balance of the species within the ecosystem depends (Holling, 1992). This idea has led to the distinction between species which are drivers and those which are passengers. The implication is that the loss of only certain species could have a massive effect on the viability of an ecosystem and the ecosystem functions that it supports. Thus only a small set of structuring processes made up of biotic and physical processes are seen as critical in influencing the structure and overall behaviour of the ecosystems; processes that are supported by different groupings of animals and plants with often complementary functions (Holling et al., 1995). A major problem, however, is that there is a significant lack of information on which species are in fact key, due to the lack of long-term ecosystem studies (Barbier et al., 1995).

It is this issue of uncertainty that lies at the heart of the biodiversity problem and results in the conclusion that at present the consequences of reductions in the number of species and specific combinations of species in terms of human welfare is often unclear. The argument that a reduction can be presumed to be negative can be presented on two counts. First, while certain species may appear to be redundant in a present ecological state, they may be of crucial importance in providing ecological functions of use to man in future evolved states. Second, genetic diversity is the basis on which the process of natural selection works, so that any reduction in biodiversity in effect hinders the evolutionary process which can be regarded as a key lifesupporting function of the biosphere. Thus, under the assumption that the environment is dynamic and will change over time, the reduction in the evolutionary potential of an ecosystem limits its ability to adapt to change and most importantly to external shocks (Common, 1995). In effect such a process can be seen as reducing the options of future generations to adapt to future changes in environmental conditions. This problem of ecological uncertainty and its implications will be further developed in Chapter 3.

An important point to stress is that, with the realization of the interconnectedness and co-dependence of the economic and environmental system as a joint system, it is evident that the joint system responds very differently to perturbation depending on where the economy and the environment are relative to the local system equilibria, as well as the characteristics of those equilibria. The dynamics of the joint system will reflect the structure of the connections between each subsystem. The existence of multiple locally stable equilibria (or basins of attraction) which are separated by unstable equilibria means that, as economic and ecological systems pass from one basin to another, the central characteristics of the system may 
undergo a profound change. If a system is in the neighbourhood of a particular unstable equilibrium or threshold, minor perturbations of its state variables may have catastrophic consequences for its structure and organization. The capacity of the system to absorb such shocks without losing stability is captured in the concept of resilience, which was briefly introduced earlier. The main implication of this characteristic is that the joint system dynamics may not be either continuous or gradual, but may flip in the face of particular shock. It is in the maintenance of the resilience of the joint system that biodiversity has been given central stage (Perrings et al., 1995). A key factor for the research presented in this book is that there is a high level of uncertainty surrounding the propensity and implications of particular phase changes, which may indeed only be recognized after they have occurred.

While the ecological and economic systems can be seen to be highly interconnected, a further source of uncertainty is derived from the fact that the two different systems do not interact evenly over time or space. Taking the latter issue, it is evident that many ecosystems cut across the structure of human society, for example the Amazon Basin spans several countries and interacts with many economic sectors (Munasinghe, 1993a). Often, therefore, the situation arises that the spatial extent of the environmental resource is more limited than the ecosystem from which it is derived. This problem of the juxtaposition of different spatial scales poses considerable questions for the sustainability debate, such as how we should evaluate changes in the scale of an economy vis-à-vis its ecological and physical context. The issue of time similarly causes difficulties for sustainability. Uncertainties over time lags in the cumulative environmental consequences of economic activity, present and future preferences and values as well as future possible substitution possibilities all pose significant constraints for meeting sustainable development objectives. As Perrings (1987) points out, it is therefore possible for components of the joint ecological-economic system to be entirely unconnected over one temporal or spatial horizon, but highly connected when viewed over some other spatial or temporal horizon.

\subsubsection{The Public Good Nature of the Environment and Uncertainty}

The public good nature of many environmental resources and the problems that this poses in relation to market failure is well documented in the environmental economics literature. It is important to note that as the environmental service or function tends towards that of a public good, familiarity decreases and information surrounding its value is often absent (Turner, 1993). Consequently the level of uncertainty is often greatest when the environmental service in question is a pure public good (see Figure 1.2). 


\begin{tabular}{|c|c|c|c|}
\hline $\begin{array}{l}\text { Pure Private } \\
\text { Goods }\end{array}$ & $\begin{array}{l}\text { Quasi Private } \\
\text { Goods }\end{array}$ & $\begin{array}{l}\text { Quasi Public } \\
\text { Goods }\end{array}$ & $\begin{array}{l}\text { Pure Public } \\
\text { Goods }\end{array}$ \\
\hline $\begin{array}{l}\text { Rivalness in } \\
\text { consumption; } \\
\text { excludability; } \\
\text { property rights, } \\
\text { market prices }\end{array}$ & $\begin{array}{l}\text { Regular } \\
\text { payments } \\
\text { in form of } \\
\text { taxes or } \\
\text { charges are } \\
\text { made to } \\
\text { finance } \\
\text { supply }\end{array}$ & $\begin{array}{l}\text { Up to some } \\
\text { capacity } \\
\text { constraint } \\
\text { (carrying } \\
\text { capacity) } \\
\text { non-rivalness } \\
\text { in consumption }\end{array}$ & $\begin{array}{l}\text { Non-rivalness in } \\
\text { consumption; } \\
\text { non-excludability, } \\
\text { or excessive } \\
\text { costs of } \\
\text { excludability }\end{array}$ \\
\hline
\end{tabular}

Source: Adapted from Turner, 1993

\section{Figure 1.2 The environmental commodities continuum}

The existence of public goods and the resulting problem of market failure lead to a gap between the market price of environmental resources and the true value to individuals and societies. It is this absence of a market price that is one of the main problems in terms of defining environmental/economic trade-offs for sustainable development objectives. One of the main causes of this gap is ignorance or uncertainty regarding the social consequences of private actions with respect to the environment. The implication of the disparity between the market price and the 'true' social costs of environmental change creates difficulties for meeting sustainable development objectives, due to uncertainty about the value of environmental change on human welfare. While the environmental economics literature has made significant developments in this field through the use of valuation, as will be shown in Chapter 2 , significant uncertainties remain which are due in part to the pure or quasi public good nature of many environmental problems such as biodiversity. This is essentially because the burden of the negative effects of uncertainty with regard to many environmental services will lie with society as a whole rather than with the individual or private company carrying out the activity.

\subsubsection{Uncertainty and the Substitutability of Ecological Functions}

The question of substitutability between the elements of man-made and natural capital in the sustainable development discourse is a core component of the uncertainty problem. Neo-classical economics tends to assume away the question of uncertainty regarding substitution policies, implying that uncertainty about the supply of essential environmental functions and services is 
not crucial, as lost environmental functions and services can be replaced by man-made capital (Common, 1995). Recent reassessments about the extent to which substitution possibilities do actually exist refute this (see: Daly, 1986; Common, 1995; Costanza 1991; Mason, 1996; Pearce et al., 1990; Pearce and Turner, 1990). Moreover, any assumptions about future technological advances and possibilities for substitution are pervaded by uncertainty. Likewise uncertainty exists about which elements constitute critical capital, due to the fact that elements that appear not to be critical currently may in fact be critical in future evolved states or unanticipated states. It is in this sense that the issue of biodiversity plays a crucial role. The role of biodiversity, in providing an opportunity set in maintaining the evolutionary potential of the joint ecological-economic system, is once again seen as crucial. There is a large degree of uncertainty over whether it is possible to substitute for the role that individual species play in the functioning of critical environmental services, in present and future unanticipated states. Subsequently the structures and processes of physical systems are seen as vulnerable to incremental human choices. These changes may not have an immediate impact, but at some spatial or temporal scale the destruction of critical ecological systems will result in direct impacts on human welfare (Norton, 1995). As Common argues, there 'remain great uncertainties regarding substitution possibilities' (Common, 1995: p. 47). While it can be conceded that some substitution possibilities do in fact exist, there remain some critical elements of natural capital, for which substitution is either not technically possible or would be too expensive, and a reduction in this critical base of natural capital in effect reduces the opportunity set on which future generations can adapt and evolve in response to stress and shock exerted on the joint ecological economic system.

The need to sustain an adequate level of natural capital means that there is therefore a need to deal with uncertainties regarding the effects of economic activity on natural capital, uncertainty over future values and preferences as well as uncertainty over the impact of future technological change. Although some argue that the question of substitutability will only be resolved empirically, many of the most important issues will only be resolved ex post. Furthermore due to the fact that many critical elements of natural capital are subject to irreversibility, by the time the uncertainty has been resolved it will be too late. It is to the issue of irreversibility and its role in the uncertainty problem that the discussion now turns.

\subsubsection{Irreversibility and Uncertainty}

The irreversibility of the damaging effects of economic activity with respect to many critical environmental functions is one of the most important prob- 
lems to be faced in the sustainable development and biodiversity discourses. Indeed it is the issue of irreversibility that necessitates the need to recognize uncertainty. If irreversibility did not in fact exist, then the question of uncertainty would not be of such paramount importance. If a mistake was made, it would be possible to reverse the effects of a particular decision, in effect giving another opportunity to roll the dice that constitutes economic activity. However, unlike financial markets, for environmental problems it may not be possible to absorb and eventually reverse previous losses. The presence of irreversibility therefore requires that sustainable development explicitly deals with the issue of uncertainty.

The importance attached to the issue of irreversibility relies on the argument that has been put forward that substitution possibilities for critical environmental services and functions are in fact limited. On this basis it is therefore important to maintain the crucial set of ecosystem properties that form the basis of the essential life supporting functions (Barbier et al., 1995). It is apparent that, if the ecosystem is pushed beyond the stage at which it is able to cope effectively with externally induced shocks, then its capacity to support the flow service may be severely diminished or removed. For example once a large area of rain forest is removed, then although over a very long period the action may be slowly reversible, the course of action over at least a time period of one generation is irreversible.

An example of an action that at present appears to be completely irreversible is the destruction of the ozone layer, as it is thought that this process cannot be reversed nor the ecosphere repaired by human effort (Hinterberger et al., 1997). In the case of biodiversity of species, the extinction of species is by definition irreversible. The argument that transformations of natural capital are for all intents and purposes irreversible is also derived from Georgescu-Roegen (1973). From an economic perspective the fact that a process may be reversible upon the expenditure of infinite energy is of no relevance if the costs are beyond the means of the decision maker. In which case in the joint ecological-economic system, it is economically irreversible. This form of irreversibility reflects the fact that the cost of rehabilitation or restoration exceeds the resources available (Perrings, 1997a).

The issue of irreversibility has attracted a wide-ranging literature in environmental economics, which, in contrast to traditional economics, does not tend to assume that resource use decisions are irreversible. The literature on irreversibility (Krutilla and Fisher, 1975; Arrow and Fisher, 1974) has tended to focus on the option of development. In particular a fundamental asymmetry has been identified that, while a decision to develop in the presence of irreversibility can not be reversed, a decision not to develop can be reversed. Thus taking the latter option leaves open the opportunity to develop in the future. While such approaches provided innovative and valuable insights into 
the problem of irreversibility, the linking of irreversibility and uncertainty is through the reliance on probability and the concepts of option and quasioption value. As Chapter 3 will go on to argue, however, this linking of irreversibility to the use of probability may only be applicable to certain cases of risk or what will be termed 'soft uncertainty'.

An additional aspect of irreversibility is in relation to the decision-making process in which, as Vickers (1994) points out, each decision moment is itself not only unique but is also embedded in the unidirectional flow of time. Shackle (1961) views such decisions as 'self destructive' in that by taking them, this forever changes the possible structure of future decisions. GeorgescuRoegen (1973) and Perrings (1987) highlight this aspect of the irreversibility with respect to the entropic processes at the heart of economic activity. Accordingly in a world governed by the laws of thermodynamics, the irreversibility of entropic processes ensures that the system will necessarily evolve through a sequence of states that are not predictable from its history. In the case of ecological-economic relationships the dynamic nature of both domains will reinforce this inherent irreversibility as an essential element of uncertainty. The impact of economic decisions on ecosystems necessarily involves changes in ecosystem structure that, however small, force acceptance of a new set of decision events which in themselves will be unique and irreversible. The uniqueness and irreversibility of the decision context and its implications for decision making under uncertainty will be looked at in more detail in Chapters 4 and 5.

\subsubsection{Equilibrium Analysis in Economic and Ecological Systems}

The issue of equilibrium and its assumptions plays a crucial role in the analysis of the economic-ecological system. A key factor in the recognition of the importance of uncertainty in ecological-economic analysis is the shift from an equilibrium framework to one which emphasizes concepts of disequilibrium. If we take the issue of equilibrium in the ecological system, it is evident that while there was a traditional tendency to use an equilibrium or static stability concept, increasing evidence has shifted the focus to that of a dynamic system. The traditional view advocated for example by Clements (1905) and Forbes (1880) was that in the case of species populations, while the populations oscillated, the oscillations were kept within bounds, tending towards an equilibrium. Although these views were firmly in the equilibrium or balance of nature camp, they did contain within them the seeds of a more modern conception of equilibrium based on the concept of resilience mentioned in earlier sections.

The weight of evidence against a static balance was such that by the middle of this century the idea of dynamic balance gained emphasis. How- 
ever, even this concept has become discredited (see Shrader-Frechette and McCoy, 1993). It has been argued by Shrader-Frechette and McCoy that it may be better to move away from employing stability terms such as 'balance', 'equilibrium', 'homeostasis' or 'stability' and towards the notion of resilience or what they term 'persistence'. The emphasis on the persistence or resilience of the system highlights the notion of a dynamic rather than a static system, in which the ecosystem or community may not in fact return to some 'normal condition' (Shrader-Frechette and McCoy, 1993). Recent contributions have reinforced the notion that ecosystems are not in fact equilibrium systems but rather dynamic systems, in which the presence of large-scale dynamics and ecosystem organization indicates that ecosystems behave in a non-linear rather than a linear manner (Norton, 1995).

While in ecology there has been a shift away from equilibrium analysis, in economics there has been a tendency to rely on the notion of static equilibria, such as those which form the basis of neo-classical models. Although there has been a recent break with this, shown by the approaches of evolutionary and ecological economic modelling (see Faucheux et al., 1996), the emphasis has remained on the use of an equilibrium framework. Equilibrium analysis, which Georgescu-Roegen sees as largely transferred from classical mechanics is essentialist or reductionist in nature and is based on the modelling of all variables and relations which are assumed to be (at least probabilistically) known and stable over time (Katzner, 1995). This reduction of uncertainty in equilibrium models to probabilistic measures will be questioned in Chapter 3.

Christensen (1996) has pointed out that the deterministic equilibrium structure not only does not admit to the interdependence of individual tastes and preferences, but perhaps more importantly for environmental problems, the neo-classical framework assumes that resources and factors of production are given, independent, and physically disconnected from other resources. In short, such a paradigm lacks a specification of the physiological and ecological structure of economic activity. In this sense an alternative non-essentialistic approach highlighted by Katzner (1995), can be extended to ecologicaleconomic issues, in which explanation is based on the idea that every conceptual phenomenon exists only as the combined result of the interactions of all other conceptual entities. Concepts cannot be said, therefore, to have single causes since they are understood to co-determine each other. This is very much in line with the notion put forward earlier of the co-evolution of economic-environmental relationships with the emphasis being on out-ofequilibrium positions in which the ideas of transition and non linearity have key roles to play (Faucheux et al., 1996). 


\subsubsection{Uncertainty and the Precautionary Principle}

This chapter has put forward the arguments that highlight the nature of uncertainty in the sustainable development problem. It is clear that the extent to which uncertainty is given importance is crucial in the way that sustainable development is defined. The argument put forward here is that if sustainable development objectives are to be met then the role of uncertainty must be recognized and tackled explicitly within the decision-making process. One of the concepts that has been put forward in relation to uncertainty in terms of sustainable development and in particular the issues of biodiversity is that of the precautionary principle.

The precautionary principle has evolved as a concept due to the presence of uncertainty and in particular irreversibility. The precautionary principle, which is similar to the principle of safe minimum standards (Ciriacy-Wantrup, 1963), in essence is a kind of insurance problem that will allow for a higher margin of error and act as a buffer against hard uncertainty. The principle states that rather than await certainty, regulators should act in anticipation of any potential environmental harm in order to prevent it from occurring and stresses the need to avoid potentially damaging outcomes (Costanza, 1994). Like sustainable development its adoption in many international environmental resolutions (for example the Rio Declaration of June 1992) has seen it evolve as a basic normative concept within many environmental discourses. The precautionary principle does recognize the problem of uncertainty as central to sustainable development by stressing our inability to predict all the future consequences of economic activity in relation to human welfare. If, as has been posited, it is the case that the dynamics of ecological systems are not in fact smooth and continuous, then the underlying notion of the precautionary principle is one that seeks to avoid the thresholds around which the resilience of the system may in fact be lost.

O'Riordan (1995) suggests a number of meanings that can be ascribed to the precautionary principle in its simplest form. ${ }^{7}$ For the purpose of this book there are two of particular interest:

1. Thoughtful action in advance of scientific proof of cause and effect based on principles of wise management and cost effectiveness. Namely that it is better to pay a little now than an awful lot later. In this sense, precaution is a receipt for action over inaction where there is a reasonable threat of irreversibility or of serious damage to life-support systems.

2. Leaving ecological space for ignorance, meaning that we should not extract critical resources, even when they are there for the taking, because we do not know what the longer-term consequences of their removal are (O’Riordan, 1995: p. 9). 
Like sustainable development, however, a number of grey areas exist in relation to the application of the precautionary principle. In particular it does not offer much in the way of guidance as to how the problem should be dealt with (Common, 1995). Foremost is the fact that, if a precautionary principle were to be applied, then it necessarily implies an opportunity cost. As Barbier et al. (1995) point out, the question soon becomes one of what things should be set aside, and whether this should be at any cost. Moreover the precautionary principle does not tell us which adverse future outcomes are the most important. Consequently, while the principle advocates a precautionary approach in the face of hard uncertainty, it does not identify how to determine the circumstances in which uncertainty is such that a precautionary approach outweighs the opportunity cost of its implementation to society.

This concern can be exemplified with the problem of biodiversity. While it may be argued that maintenance of biodiversity is an essential element in sustaining the opportunity set for future generations in the face of perturbations to the ecological-economic system, it has also been the case that biodiversity loss, through natural resource selection such as in agriculture, has in many cases improved human welfare (Perrings, 1994). Clearly, therefore there is a need for a means of assessing the trade-offs between adopting a precautionary approach to uncertainty about the environmental implications of development and the social benefits of development. It is the need to deal with this type of uncertainty within a decision-making framework that will be highlighted in the following chapters.

\subsection{A RESEARCH FRAMEWORK}

This chapter has stressed the crucial role that uncertainty plays in the research context of the sustainable development and biodiversity discourse. Identifying uncertainty as a crucial factor immediately poses a number of challenges for traditional scientific research due to the very nature of the uncertainty problem at hand as well as the subjective nature of human behaviour in the face of uncertainty. Indeed rather than reducing the level of uncertainty surrounding the ecological-economic system, it should be realized that the progress of science has in fact uncovered more rather than less uncertainty (Costanza, 1994). As a consequence, given that for many environmental problems a large number of the facts are uncertain, and that value will be in dispute, it is often 'soft' scientific information which will end up serving as the inputs for 'hard' policy decisions to be made.

In this scheme of things it may be, as Norton (1995) argues, time to 'loosen the intellectual hinges, to try many different models and engage in 
disciplinary crossovers to increase communication across disciplines' (Norton, 1995: p. 122).

Funtowicz and Ravetz (1991) argue that such a new approach might be termed 'post normal science' in which scientific method would no longer necessarily imply anything about the precision of the results achieved, and in which the concept of the scientific explanation and of what constitutes a good decision is being changed. What it does imply is a forum for open inquiry which has no preconceived answers and which is aimed at exploring the extent of our knowledge and the magnitude of our ignorance (Costanza, 1994). By creating a new relationship between retaining the basic principles of scientific method and adapting to the reality of uncertainty, a new place for science in human affairs can be fashioned. It is this philosophy that will underlie this research.

\subsubsection{Aims, Hypotheses and Outline of Research}

Although the topic of uncertainty and accompanying research framework would suggest the difficulty of making testable hypotheses which can be verified as true or false, it will be useful to outline some broad hypotheses or research questions which this book aims to answer and to formulate the nature of the problem to be addressed. There are four main hypotheses or concerns which are the focus of the research presented in this book:

1. Existing conceptualizations of uncertainty and in particular environmental uncertainty do not reflect the full range of uncertainty faced in decision making.

2. By recognizing that there are in fact a number of different modalities of uncertainty, it is argued that the use of utility maximizing models, based on the notion of probability, do not adequately deal with the range of environmental uncertainty faced by decision makers.

3. The Shackle model better explains the way that decisions are made in the face of uncertainty and in the context of improving the procedural rationality of the decision-making process can be fruitfully employed in a prescriptive sense.

4. Building on this, it is hypothesized that the Shackle model can be operationalized in a real world decision context, as is done in a case study which focuses on the Belize Southern Highway Project.

As well as the theoretical contribution that this book aims to make, a core element is the application of the Shackle model to environmental uncertainty in respect of a road development project in Belize. The case study not only demonstrates examples of the type of uncertainty that is faced in such decisions, 
but also demonstrates the application of the Shackle model as a means of explaining decision making in the face of hard uncertainty. As well as developing a model of decision making under uncertainty, the interviewing of decision makers and the collection of additional information provides a rich source of qualitative data, in which to contextualize the application of the model. The case study also provides the practical basis on which the model is used to develop a prescriptive decision-making framework capable of explicitly dealing with environmental uncertainty.

This chapter has given an introduction to the problem of uncertainty in the context of sustainability and has outlined the research framework that is to be adopted. In the following chapter the decision-making context of cost-benefit analysis, and the valuation of environmental resources will be briefly reviewed. After that, Chapter 3 takes the important step of conceptualizing uncertainty at an epistemological level before providing a framework for defining the different modalities of uncertainty. Using this framework a theoretical basis is developed, highlighting the dimensions of environmental uncertainty. Building on this, a critique of the use of probability in environmental decision making in the face of hard uncertainty will be made, and the need for an alternative framework to that of expected utility forwarded (Chapter 4). In Chapter 5 the Shackle model will be introduced as an innovative alternative in the context of environmental uncertainty and its application in decision making analysed. Chapter 6 introduces the case study of the Belize Southern Highway and Chapter 7 describes the methodology adopted to carry out the fieldwork. Chapter 8 assesses whether the evidence suggests that the model is useful in explaining decision making under uncertainty. Chapter 9 looks at the way that uncertainty is handled at a more general level in the decision-making process and develops a normative framework for dealing adequately with uncertainty. In Chapter 10 conclusions are drawn.

\section{NOTES}

1. Hard uncertainty can be defined at this stage in terms of situations in which a) the set of possible actions or future states is unknown or b) where an exhaustive set of future states can be specified, the probability distribution for such outcomes is unknown or not fully definable. A more comprehensive definition of hard uncertainty and the different modalities of uncertainty is left until Chapter 3.

2. Soft uncertainty or risk is used to define situations where 1) the set of all possible outcomes of an action are known and 2) the probability distribution of all possible outcomes is also known (see Section 3.2.1).

3. The only exception was its use by Perrings (1989) in a theoretical paper, see Chapter 3.

4. The report is officially titled Our Common Future (World Commission on Environment and Development, 1987) but derived its popular title from the name of the chairman of the Commission, Gro Harlem Bruntland.

5. While attention is focused on the economic and ecological sustainability aspects of this 
framework, it is important to emphasize the important contribution of the socio-cultural view to the sustainability debate. (See for example O'Connor, 1988, 1989; Escobar, 1996; Oslender, 1997).

6. Figure 1.1 is based on the structure of the diagram contained in Munasinghe (1993b: p. 2) but has been subsequently adapted to take into account the author's own views.

7. Also see O'Riordan and Cameron (1994) for greater detail on the precautionary principle. 